\title{
Extraction of Blood Vessels from Retinal Image using Adaptive Morphology
}

\author{
Rajat Suvra Nandy \\ Dept. of Comp. Sc. \& Engg. \\ Birla Institute of Technology \\ Mesra, India
}

\author{
Rohit Kamal Chatterjee \\ Dept. of Comp. Sc. \& Engg. \\ Birla Institute of Technology \\ Mesra, India
}

\author{
Avijit Kar, PhD \\ Dept. of Comp. Sc. \& Engg. \\ Jadavpur University \\ Kolkata, India
}

\begin{abstract}
In this paper a new algorithm is proposed for segmentation of the retinal blood vessels in the ophthalmoscopic images using Mathematical Morphology based technique named as Adaptive Line Structuring Element (ALSE). The ALSE is rotated to adjust with the curvature of the blood vessels which ensure that each part of the vessel components remains connected and followed by changing the size of the line structuring element that adaptively discards non-vessel like objects in a fundus image. This forms a Scale-Space that extracts the blood vessels structure in retina successfully from finer scales to coarser scale without much loss of data. For the purpose of evaluating performance of proposed algorithm two well-known criterion function namely Structural Similarity (SSIM) index and Figure of Merit (FOM) index are used for comparing the results. The average value of SSIM and FOM indicates better performance of the proposed algorithm than other recent methods.
\end{abstract}

\section{General Terms}

Medical Image Processing, Diabetic Retinopathy, Ophthalmoscopic Image.

\section{Keywords}

Adaptive Mathematical Morphology, Adaptive Line Structuring Element, Retinal Images, Curvature Scale-Space.

\section{INTRODUCTION}

The Diabetic Retinopathy (DR) is very common complication of the chronic diabetic patients, but diagnosis and treatment of DR requires intervention of specialized experts with large amount of cost and time. Prognosis of diabetic retinopathy is related to accurate detection of pathological changes in blood vessels structure of retina. Hence, today one of the challenging task is to automatic extraction of blood vessels from retinal images (digital Fundoscopic images). Likewise, automatic extraction of blood vessels may help to detect different other type of ophthalmic diseases, such as retinal hemorrhage of different causes including trauma, geriatric retinal atrophy, etc. Quick and early detection of different type of pathology on the retina may save many people in the world from blindness.

Earlier techniques for extraction blood vessels from retinal image can be classified into two types [1]. First one is window-based [2], [3] and other is Tracking-based [4], [5], [6]. Window based approaches explore the features of a pixel's in the neighborhood and highlights those pixels which matches with a given model. Most of the models are usually based on classical line detection methods found in the literature. In [2], the cross section of a blood vessel is detected by a Gaussian shaped curve. After that the matched filters of twelve directions are applied to highlight the vessels structure.

On other hand, tracking-based approach uses a blood vessel profile model. Here the tracing are started from some initial points and it gradually follows a track which is best matched with the profile model. In [4], the tracing begun from the papilla. After that it is traced with respect to the continuities of location, twist, density and diameter of the vessels structure. The tracing is upgraded by applying the fuzzy model of vessel structure profile [6]. Another enhancement is achieved by applying the local region-based threshold searching [7]. In fact, this method is a composition of the window-based and as well as tracing-based methods.

The thickness of retinal blood vessels can fluctuate from large size to small size and also the local contrast of the blood vessels may be near to its background, particularly in corrupt ocular fundus. So, the automated extraction of blood vessels is actually challenging task and there is a scope to improve the existing algorithms.

In this paper a novel technique is proposed which automatically extracts the blood vessels structure from retinal image by rotating a line structuring element of different size over the image. This line structuring element detects the curvatures with minimal loss of shape and structures of the vessels and a curvature scale space is obtained in this process. A suitable automated threshold is determined to eliminate noise like structures from the resultant image. Non-destructive extraction of blood vessels of retina is a prior condition for correct diagnosis of many ophthalmic diseases. Thus proposed method will facilitate to detect quickly and efficiently the different ophthalmic diseases which deforms the structure and shape of vessels of retina without much intervention of an expert.

Proposed technique focus on an unsupervised scheme i.e. no previous knowledge of the characteristics of the image is required. The algorithm has three stages major steps. Firstly, the unwanted structures are removed by using morphological opening and closing. Next, an Adaptive Line Structuring Element (ALSE) is generated and using this ALSE the blood vessels are extracted from the retinal image. The ALSE appropriately adapts the local vessel structure of different size and curvature. Lastly, a statistical threshold is devised to differentiate the blood vessels from background noise. The ultimate result produces a clear binary image of retinal blood vessels structure without loss of details.

Rest of the paper is organize as follows: Section 2 gives a very brief account of some basic mathematical morphology operations for reference purpose; Section 3 elaborates the proposed algorithm in detail; Section 4 presents the results of 
the algorithm applied on the test images with comments on the values of Structural Similarity (SSIM) index and Figure of Merit (FOM) and also a comparative study with other recent methods. Lastly in Section 5, we conclude by summarizing the main proposal of this paper.

\section{BASIC MORPHOLOGICAL OPERATIONS}

This section gives an overview of some basic operations in gray-level mathematical morphology [8] that are used in the proposed algorithm.

A two dimensional image $f(x, y)$, where $(\mathrm{x}, \mathrm{y}) \in \mathbb{Z}^{2}$, and operated by a structuring element $b(x, y)$ where $(\mathrm{x}, \mathrm{y}) \in D_{b} \subseteq \mathbb{Z}^{2}$ is assumed in the following discussion. The definition of basic gray-level mathematical morphology operations are described below for reference purpose.

\section{Dilation:}

The Grayscale Dilation is defined as

$$
\begin{aligned}
\delta_{b}= & \max \left\{f\left(x-x^{\prime}, y-y^{\prime}\right)+b\left(x^{\prime}, y^{\prime}\right)\right\} \\
& \text { where }\left(x^{\prime}, y^{\prime}\right) \in D_{b}
\end{aligned}
$$

Erosion:

The Grayscale Erosion is defined as

$$
\begin{aligned}
\varepsilon_{b}= & \min \left\{f\left(x+x^{\prime}, y+y^{\prime}\right)-b\left(x^{\prime}, y^{\prime}\right)\right\} \\
& \text { where }\left(x^{\prime}, y^{\prime}\right) \in D_{b}
\end{aligned}
$$

Where, $D_{b}$ domain of $b$, and $f(x, y)$ is assumed to equal $+\infty$ outside domain of $f$.

$$
\begin{aligned}
& \text { Opening: } \\
& \gamma_{b}(f)=\delta_{b}\left(\varepsilon_{b}(f)\right)
\end{aligned}
$$

Closing:

$$
\phi_{b}(f)=\varepsilon_{b}\left(\delta_{b}(f)\right)
$$

\section{Top-Hat Transformation:}

$$
T_{\text {hat }}(f)=|\gamma(f)-f|
$$

The above operations are applied in different combinations with respect to the different structuring element size and shape in order to remove or restore local structures of blood vessels of retina. The notations explained above are used in our proposed algorithm. Disc shaped and line structuring elements (ALAS) mainly used in the proposed algorithm.

\section{PROPOSED VESSEL SEGMENTATION TECHNIQUE}

\subsection{Preprocessing of Fundus Image}

In the first step where the colored fundus image $\left(I_{R G B}\right)$ is converted into a gray-scale fundus image $\left(I_{\text {gray }}\right)$ to help the blood vessels separation and to decrease the computational time. This has been observed that, the green channel of the RGB retinal image contains the maximum information about the blood vessels and the red channel contains the pathological data [9]. So, the RGB fundus image $\left(I_{R G B}\right)$ is converted into grey-scale image using Craig's formula (6) given below

$$
I_{\text {gray }}=0.3 \times R+0.59 \times G+0.11 \times B
$$

where, $I_{\text {gray }}=$ Gray scale image, $R=$ Red Channel, $G=$ Green Channel, $B=$ Blue Channel of the given fundus image $\left(I_{R G B}\right)$ and 59\% importance is given to the green channel, because it has the maximum contrast in between blood vessels. The background of retinal images is rather saturated by the red channel and the blue channel is rather dark [10]. The red channel in the image has spread intensity level in the location where the green channel has very low contrast difference. It gives extra variation between the blood vessels and optic disc of retinal image. The most unsupervised methods [11], [2], [12] use the green channel to extract the vessel structure.

\subsection{Initial Elimination of Background}

The same technique that is found in [13], [14] is followed in this step to extract the vessel like shape. It is already known from literature that thickness of the retinal blood vessels ranges between 3 pixels to 7 pixels [18]. A disc shaped structuring elements $S_{r 3}$ and $S_{r 8}$ having radius 3 and 8 pixels are used successively to eliminate the non-vessel like structures. At first the morphological opening (3) is executed on the gray-scale retinal image $I_{\text {gray }}$ with the structuring element $S_{r 3}$ to remove the non-vessel like objects having size smaller than 3 pixels and the output image we denote by $I_{o p}$. This operation is defined as

$$
I_{o p}=\gamma_{S_{r 3}}\left(I_{\text {gray }}\right)
$$

Now, the morphological closing (4) with the structuring element $S_{r 8}$ is applied on the image $I_{o p}$ to connect the objects which are less than 8 pixels and output image is $I_{\text {op_cl }}$. This operation is defined as

$$
I_{o p_{-} c l}=\phi_{S_{r 8}}\left(I_{o p}\right)
$$

The structuring element $S_{r 8}$ is applied for the above mentioned morphological closing operation, having the knowledge that the blood vessels have maximum radius of 7 pixels.

This Opening and Closing operation results an image $I_{o p \_c}$ with no vasculature. So, finding the differences between the original gray-level image $\left(I_{g r a y}\right)$ and $I_{o p_{c} c l}$ extracts the vessel like structure by eliminating background. This operation is well known morphological top-hat transformation (5) as described below

$$
I_{r s t}=\left|I_{o p_{-} c l}-I_{\text {gray }}\right|
$$

By this process the retinal blood vessels can be extracted from the fundus image, but it retains the objects having size in between 3 to 7 pixels which may not be the part of retinal blood vessels.

\subsection{Generate an Adaptive Structuring El- ement}

The unwanted non-vessel like objects are retained in the fundus image when the morphological closing is used with the structuring element $S_{r 8}$ according to (8). The fundus image contains vessels which are elongated structures. So, the disc shaped structuring element is incapable in finding the vessel like structure in the retinal image. Hence, the image $I_{r s t}$ has many non-vessel structures and those structures do not contribute to the vasculature. The local structure of blood vessels can be approximated as consisting of small straight lines, whose width is one pixel only and our proposed technique is based on this perception. A one pixel thick line that changes its size followed by rotation which collects the maximum response in the neighborhood is so-called Adaptive 


\section{\begin{tabular}{l|l|l|l|l|l|l|}
\hline 1 & 1 & 1 & 1 & 1 & 1 & 1 \\
\hline
\end{tabular} Origin of $\boldsymbol{S}_{\theta^{\circ}}{ }^{\circ}$}

(a)

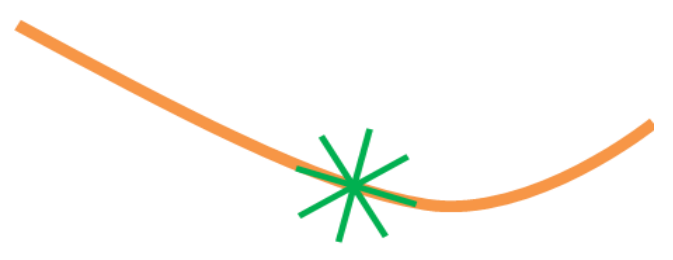

(c)

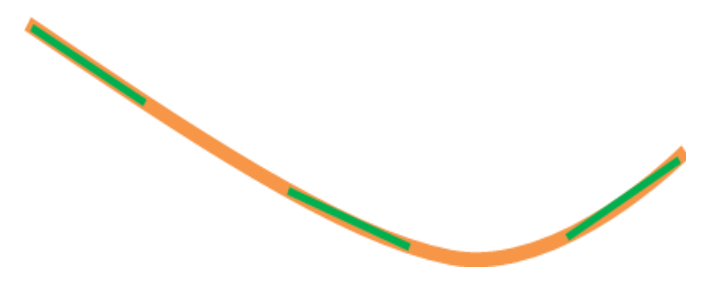

(d)

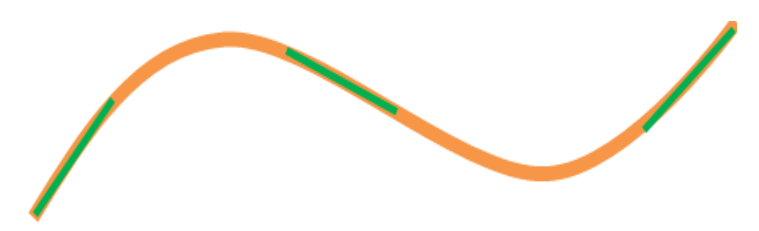

(e)

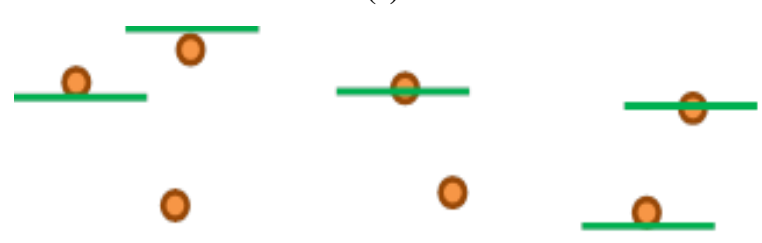

(f)

Fig. 1: (a) Adaptive Line Structuring Element $\left(\mathbf{S}_{0^{\circ}}(\mathbf{7})\right)$ with size 7 pixels, (b) Rotation of $\operatorname{ALSE~} S_{\theta^{\circ}}(7)$, (c) The rotation of LSE with respect to its origin in image domain gives the maximum response and the resultant image is formed (Green lines are line structuring element and Orange Lines are patterns in image), (d)

Curvature detection, (e) Vessels detection, (f) Noise like element elimination

Line Structuring Element (ALSE). The Adaptive Line Structuring Element $\left(S_{\theta^{\circ}}(i)\right.$, where, $i \in \boldsymbol{Z}$ and $\theta^{\circ}$ is given angle of rotation) contains two parameters Size $(i)$ and Angle $\left(\theta^{\circ}\right)$. The size $i$ defines the length (number of pixels) of the line structuring element, whereas the angle $\left(\theta^{\circ}\right)$ defines the orientation of the ALSE. The line structuring element with size $i$ is a one dimensional array with the size of $i$ pixels having all $1 \mathrm{~s}$ as shown in Figure 1(a). The origin of the adaptive line structuring element $\left(S_{\theta^{\circ}}(i)\right)$ is at $(i / 2+1)$ th pixel position of the array. The ALSE overlaps on each pixel of the fundus image with respect to the position of its origin.

\subsection{Extraction of Retinal Blood Vessels using Line Adaptive Structuring Element}

The Morphological opening is an anti-extensive property and this operation does not generate any new structures in the image. Hence, the morphological opening on the resultant fundus image $I_{r s t}$, using the adaptive line structuring element $\left(S_{\theta^{\circ}}(i)\right)$ is applied by increasing its size in each step. The size of $S_{\theta^{\circ}}(i)$ varies from 1 pixel to $n$ pixels and for each value of " $i$ ", the angle $\left(\theta^{\circ}\right)$ of $S_{\theta^{\circ}}(i)$ varies from $0^{\circ}$ to $180^{\circ}$ with the increment of $10^{\circ}$ in the experiments conducted below. The image pixel has been taken at that direction where the maximum intensity value is found after applying this operation. The summary of these operations are shown by in the following equations (10), (11), (12), and (13).

$$
\begin{aligned}
& I_{\theta(j+1)}(i)=\gamma_{S(i, \theta(j))}\left(I_{r s t}\right) \\
& \theta(j+1)=\theta(j)+10^{\circ}
\end{aligned}
$$

Here, $I_{\theta(j+1)}(i)$ represents the resultant image after Opening the image $I_{r s t}$ by the line structuring element $S(i, \theta(j))$, where $i$ changes from 1 pixel to $n$ pixels and $\theta(j)$ changes from $0^{\circ}$ to $180^{\circ}$ with the increment of $10^{\circ} . \mathrm{j}^{\mathrm{N}}$ varies from 1 to $180^{\circ} / 10^{\circ}$ $(=18)$.

$$
\begin{array}{r}
I_{\max }(i)=\max \left(I_{\theta(j)}(i)\right) \\
\text { where, } j=1 \text { to } 18
\end{array}
$$

Now, we generate adaptability by changing the size $(i)$ of the line structuring element from 1 pixel to $n$ pixels. This approach is shown by using (13).

$$
\begin{aligned}
& I_{\theta(j)}(i+1)=\gamma_{S(i+1)}\left(I_{\max }(i)\right) \\
& \text { where, } I_{\theta(j)}(1)=I_{\max }(1) \text { and } i=1 \ldots n .
\end{aligned}
$$

Length of structuring element $(S(i+1, \theta(j)))$ incrementally increased to be applied on the previous image $\left(I_{\theta(j)}(i)\right)$. The operation progresses from small to large size adaptive line structuring element (ALSE) by using the image found in the previous step. The consecutive morphological opening holds the curvature of the retinal blood vessels for the definite size.

For a specific size of adaptive line structuring element $r$ (where, $r \in \boldsymbol{Z}$ ) we have the appropriate gray-scale retinal blood vessels structure $\left(I_{\text {gray }}(r)\right)$ that removes all the undesirable objects and keeps the retinal blood vessels intact. So, $I_{\text {gray }}(r)$ is the image which retains the retinal blood vessels structure of size upto $r$. This operation is described by

$$
I_{\text {gray }}(r)=\gamma_{S(r, \theta(j))}\left(I_{r s t}\right)
$$

\subsection{Separating the Vessel Structure from Background}

The resultant retinal image $I_{\text {gray }}(r)$ contains only vessel like structures. Now, to segment the blood vessel from its background a suitable threshold is required to be applied on $I_{\text {gray }}(r)$.

At first, to increase the contrast of an image, we raise each pixel of $I_{\text {gray }}(r)$ to the $k^{\text {th }}$ power (where $k$ is a real number). The procedure is described as

$$
I_{\text {enh }}(x, y)=\left(I_{\text {gray }(r)}(x, y)\right)^{k}
$$


After that to extract the retinal blood vessel structure from the enhanced resultant retinal image $\left(I_{e n h}\right)$, we formulate a statistical threshold to convert the $I_{e n h}$ into a binary image given by

$$
I_{\text {vessel }}= \begin{cases}1 ; & \text { where }, I_{\text {enh }}>\left(\mu\left(I_{\text {enh }}\right)+\eta \times \sigma\left(I_{\text {enh }}\right)\right) \\ 0 ; & \text { where, } I_{\text {enh }} \leq\left(\mu\left(I_{\text {enh }}\right)+\eta \times \sigma\left(I_{\text {enh }}\right)\right)\end{cases}
$$

where, $I_{\text {vessel }}$ is the binary image with desired resultant retinal blood vessels structure. The mean and standard deviation of the enhanced retinal image $\left(I_{e n h}\right)$ is represented by $\mu\left(I_{e n h}\right)$ and $\sigma\left(I_{e n h}\right)$ respectively, and " $\eta$ " is an adjustable parameter.

The resultant retinal blood vessels structure $\left(I_{\text {vessel }}\right)$ is a binary image, having value one for retinal vascular structure and zero for the background pixels.

\section{EXPERIMENTAL RESULTS \& DISCUSSIONS}

The proposed Adaptive Mathematical Morphology based filter is tested on the freely available images found in Drive database [16]. In the Drive database, for each retinal image there is a corresponding mask image to segment the region of interest and also a manually segmented (ground truth) image of size $584 \times 565$ pixels. At first, the mask is applied on each image to extract the fundus region. Then, method described in equation (6) is used to convert the image into a grayscale image and result is shown in Figure 2(b). On this grayscale image morphological opening using a disc shaped structuring element $\left(\boldsymbol{S}_{r 3}\right)$ is applied as given by the equation (7). The output image $\left(I_{o p}\right)$ shown in Figure 2(c) where all the objects in $I_{\text {gray }}$ which are more than 3 pixels have been removed. Then $I_{o p}$ morphologically closed using the structuring element $S_{r 8}$ as mentioned in equation (8). The resultant image $I_{o p_{-} c l}$ is shown in the Figure 3(a) and it contains all the connected objects which are less than 8 pixels.

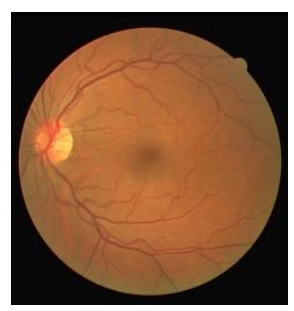

(a)

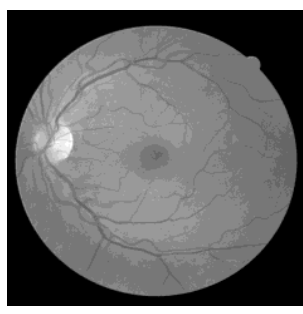

(b)

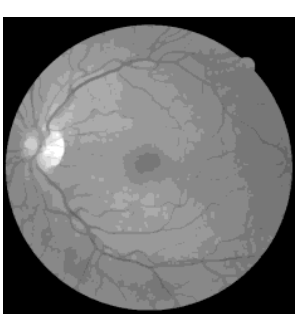

(c)

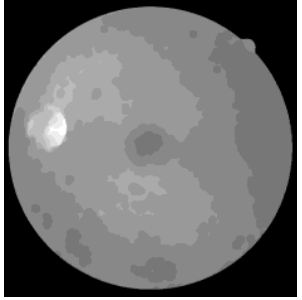

(a)

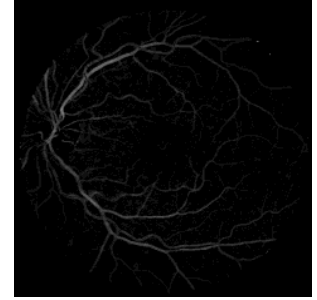

(b)
Fig. 3: (a) Output Image $\left(I_{o p-c l}\right)$ ) after Closing of $I_{o p}$ by structuring element $S_{r 8}$ (b) Difference between $I_{o p-c l}$ and $I_{\text {gray. }}$.

Now an Adaptive Line Structuring Element (ALSE) with variable size is applied and followed by rotating the structuring element in increment of $10^{\circ}$ from $0^{\circ}$ to $180^{\circ}$. By this method curvature scale-space of gradually increased size is created. The maximum response along the direction has been taken as the resultant pixel. The experiments done in this paper, the initial step started with a line structuring element of size 3 pixels. Then the second step length of line structuring element increased to 4 pixels and applied on previous scaled image. Successively we continue to additional scaling as

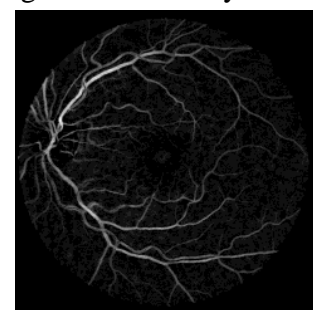

(a)

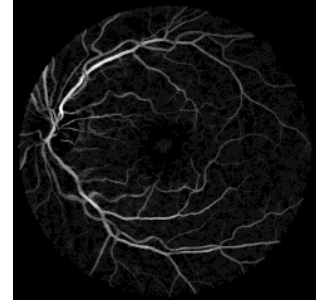

(c)

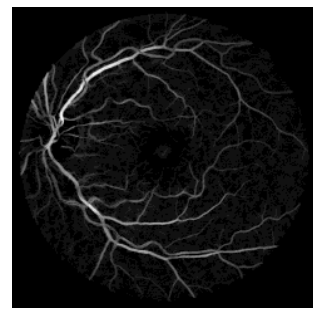

(e)

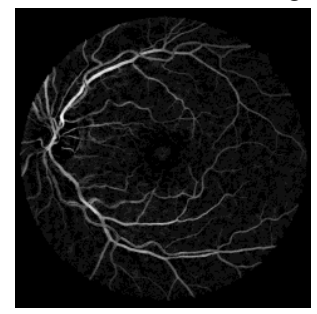

(b)

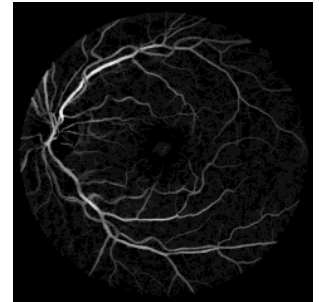

(d)

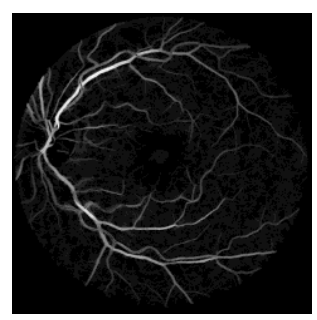

(f)
Fig. 2: (a) 584 $\times 565$ pixels Fundus Image (b) Preprocessed grayscale Image $\left(I_{\text {gray }}\right)$ and (c) Output $\operatorname{Image}\left(I_{o p}\right)$ after Opening of $I_{\text {gray }}$ by $S_{r 3}$

Next, the difference between $I_{\text {op_cl }}$ and $I_{\text {gray }}$ is shown in the Figure 3(b) and the output image is $I_{r s t}$ which is constructed using (9). $I_{r s t}$ contains only the objects of size between 3 to 7 pixels as mentioned in [13], [14]. 
with taking into account of the orientation of the blood vessels.

The big advantage of this technique is that the small blood vessels are preserved, because initial starting scale is small enough (3 pixels) and gradually the length of the line structuring element is increased.

We have changed the length of the line structuring element ranging from 3 pixels to 30 pixels. The experimental results with the changing the size of structuring element are shown in Figure 4.

The Figure 4, shows the different images after applying the Adaptive Line Structuring Element on grayscale image $I_{r s t}$ resulted in last step, with size such as (a) 5 pixels (b) 7 pixels, (c) 11 pixels, (d) 13pixels, (e) 15 pixels, (f) 20 pixels.

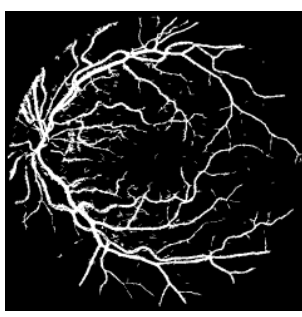

(a)

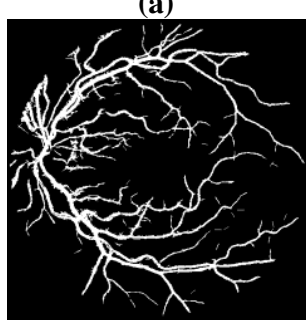

(c)

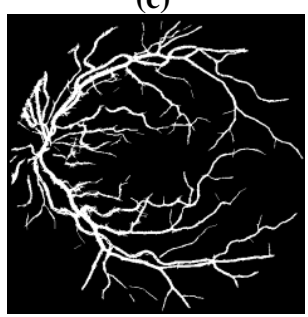

(e)

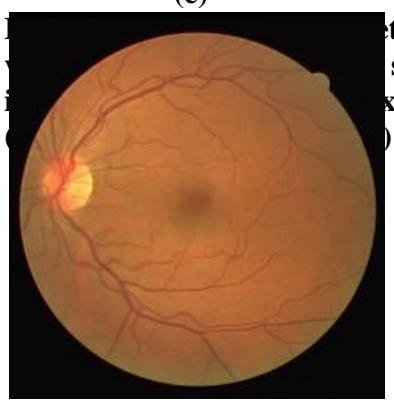

(a)

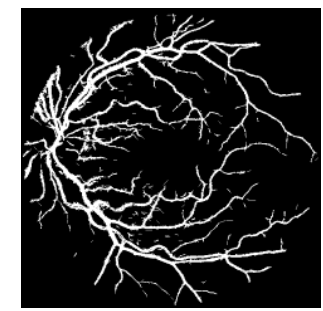

(b)

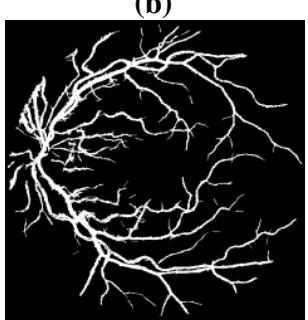

(d)

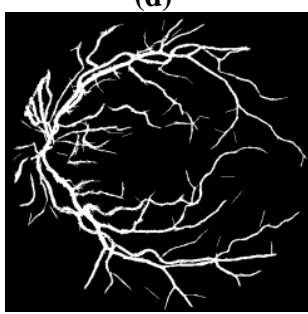

(f)

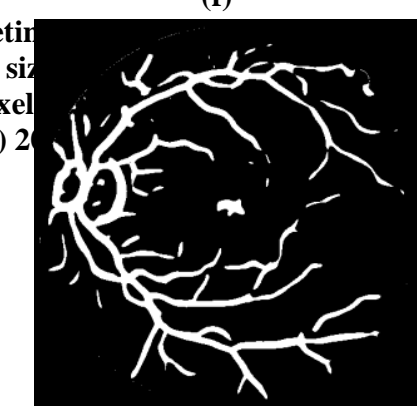

(b)
Fig. 6: (a) $584 \times 565$ pixels Fundus Image (b) The output image formed after applying Salem et al. [10] method on the image of DRIVE database given in 6(a)
To remove the background we apply the statistical threshold given by equation (16), which is the resulted binary image of the retinal blood vessels $\left(I_{\text {vessel }}\right)$ from the image $I_{e n h}$. The results are shown in Figure 5.

In Figure 5(a) we have a binary retinal blood vessels structure with respect to its gray-scale image which is shown in Figure 4(a) and it contains some background noise. But when the length of the ALSE is increased then the noises are eliminated gradually, which are shown in Figure 5(b-f). Visually the best results for binary retinal blood vessels structure are obtained when the length of the ALSE ranges from 15 pixels to 20 pixels.

To measure the accuracy of our proposed method we apply the technique on all fundus images of DRIVE database. Then we compute the Structural SIMilarity (SSIM) indexes among our resultant images and its corresponding manually segmented images, which contains vessels structure which are sketched by experts as provided in the DRIVE database.

The formula of SSIM index [15] between two images $x$ and $y$ of common size $M \times N$ is as follows

$$
\operatorname{SSIM}(x, y)=\frac{\left(2 \mu_{x} \mu_{y}+c_{1}\right)\left(2 \sigma_{x y}+c_{2}\right)}{\left(\mu_{x}{ }^{2}+\mu_{y}{ }^{2}+c_{1}\right)\left(\sigma_{x}{ }^{2}+\sigma_{y}{ }^{2}+c_{2}\right)}
$$

Where, $\mu_{x}$ and $\mu_{y}$ denotes the average of $x$ and $y$ respectively, $\sigma_{x}{ }^{2}=$ the variance of $x, \sigma_{y}{ }^{2}=$ the variance of $y, \sigma_{x y}=$ the covariance of $x$ and $y, c_{1}$ and $c_{2}$ are two variables to stabilize the division.

After calculating SSIM index of the output images of our proposed algorithm with respect to its corresponding manually segmented images, we plot the graphs with respect to different scales, which are shown in Figure 7(a to d). The graphs show the change of SSIM index versus length of adaptive line structuring element. The best results of SSIM index of the resultant images of our proposed method can be found for the length of ALSE between 17 to 22 pixels, which validates the visually found results.

Also to reaffirm our observation, we use the Figure of Merit (FOM) criterion function as proposed by Pratt [17] to analyze the errors related in edge detection procedure. Formula of FOM is given as

$$
F O M=1 / I_{N} \sum_{i=1}^{I_{A}} \frac{1}{1+a d^{2}}
$$

Where $I_{N}=\max \left\{I_{I}, I_{A}\right\}$ and also $I_{I}$ is the number of ideal and $I_{A}$ is the number of actual edge map points. The distance of separation of the actual edge point normal to the line of ideal 


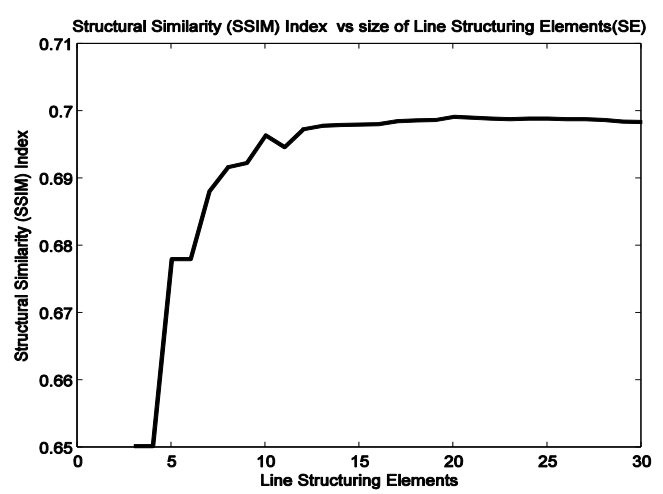

(a)

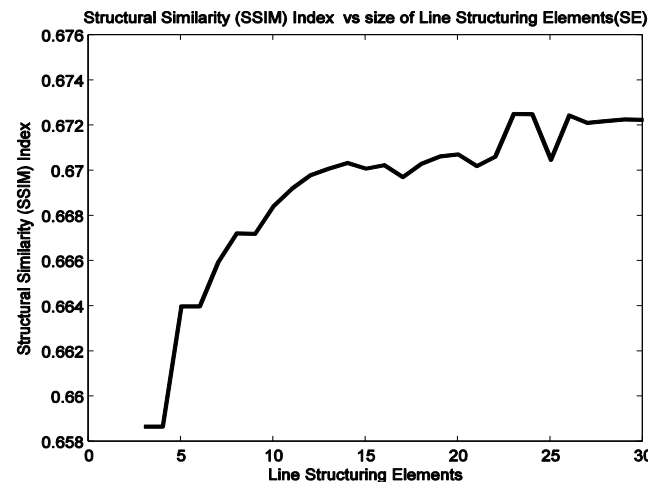

(b)

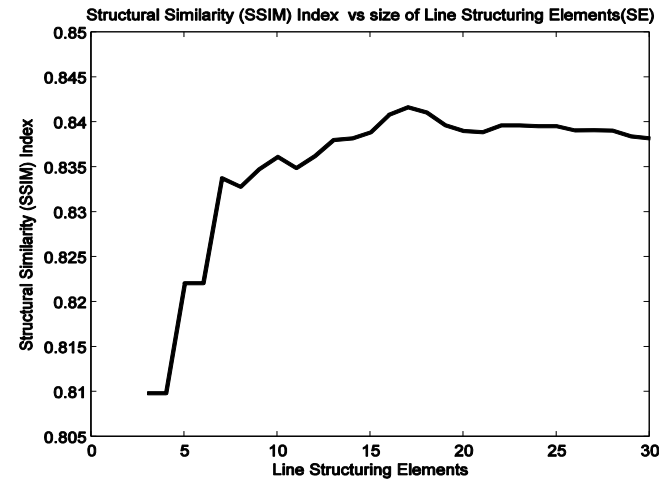

(c)

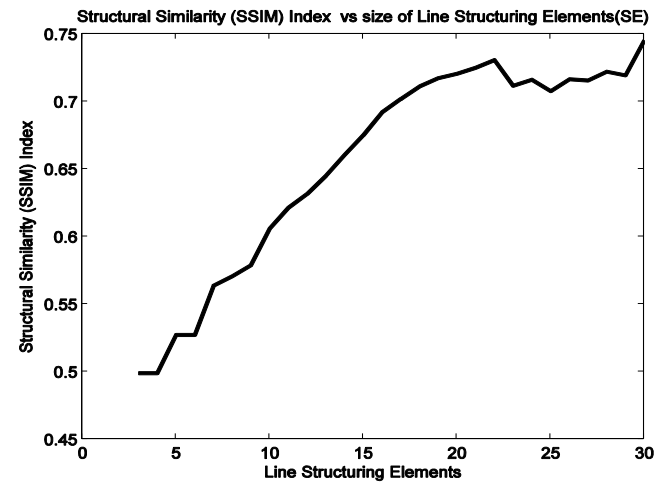

(d)

Fig. 7: Computation of Structural Similarity (SSIM) Index on the fundus images (a) 01_test.tif, (b) 05_test.tif, (c) 15_test.tif , and (d) 20_test.tif of DRIVE Database with respect to the change of the length of ALSE edge points is given by $d$. Here, ' $a$ ' is a scaling constant taken as $1 / 9$ (according to the Pratt's original work).

We have compared our proposed Adaptive Mathematical Morphology based filter with the recently proposed methods by Salem et al. [10] and Samanta et al. [14] and the result is compared in Table 1. For visual comparison, Figure 6(b) shows the output images after applying Salem et al. [10] algorithm on the same image of DRIVE database.

Table 1. shows the average values of both SSIM and FOM indexes of the outcome images and its corresponding manually segmented images for above mentioned three methods for all 40 images found in the DRIVE database.

Table 1. Comparison of three methods of SSIM and FOM

\begin{tabular}{|c|c|c|}
\hline Methods & $\begin{array}{c}\text { Average SSIM } \\
\text { index value }\end{array}$ & $\begin{array}{c}\text { Average FOM } \\
\text { index value }\end{array}$ \\
\hline Salem et al. [10] & 0.4482 & 0.2722 \\
\hline $\begin{array}{c}\text { Samanta et al } \\
\text { [14] }\end{array}$ & 0.4606 & 0.2128 \\
\hline $\begin{array}{c}\text { Our Proposed } \\
\text { Algorithm }\end{array}$ & 0.7418 & 0.5569 \\
\hline
\end{tabular}

Table 1. clearly shows that, SSIM index and FOM index respectively of the Adaptive Mathematical Morphology based filter method evaluated is higher than two other recently proposed methods of retinal blood vessel segmentation on publicly available standard DRIVE database images.

\section{CONCLUSIONS}

This paper proposes a novel technique to extract the blood vessels structure from retinal fundoscopic image using adaptive mathematical morphology. Anti-extensive property of the morphological operators used ensures that no new objects (artefacts) are introduced in the images. It does not depend on any intrinsic property of the given image such as, thickness or density of the vessels. The Structural Similarity (SSIM) indexes and the Figure of Merit (FOM) indexes of the resultant images with respect to the binary images which are given in the manual-1 and manual-2 in the DRIVE database are in average of 0.7418 and 0.5569 respectively. These results are significantly better compared to other recently proposed methods. The experimental results demonstrate that the proposed adaptive morphological technique is good enough to detect the small and large blood vessels structure simultaneously. This method is also useful to remove the noise and enhance the vessel structure. So, the blood vessels with low contrast may be deleted accurately. The only drawback remaining with this technique is to decide the number of iteration required for correct preservation of the blood vessels. This problem will be addressed in our future work.

\section{ACKNOWLEDGMENTS}

The authors are very much grateful to the authority who maintains the "DRIVE" Database for providing fundus images which has required to test the robustness of the Our Proposed Algorithm. 


\section{REFERENCES}

[1] Li, Q., You, J., Zhang, L. and Bhattacharya, P. 2006. Automated Retinal Vessel Segmentation Using Multiscale Analysis and Adaptive Thresholding. IEEE Trans. Image Analysis and Interpretation, pp.139-143.

[2] Chaudhuri, S., Chatterjee, S., Katz, N., Nelson, M. and Goldbaum, M. 1989. Detection of blood vessels in retinal images using two-dimensional matched filters. IEEE Trans. Med. Imag., vol. 8, pp. 263-269.

[3] Pappas, T. and Lim, J. 1988. A new method for estimation of coronary artery dimensions in angiograms. IEEE Trans. Acoust., Speech, Signal Processing, vol. 36, pp. 1501-1513.

[4] Sun, Y. 1989. Automated identification of vessel contours in coronary arteriograms by an adaptive tracking algorithm. IEEE Trans. Med. Imag., vol. 8, pp. $78-88$.

[5] Tamura, S., Okamoto, Y. and Yanashima, K. 1988. Zero crossing interval correction in tracing eye-fundus blood vessels. Pattern Recognition., vol. 21, no. 3, pp. 227-233.

[6] Tolias, Y. and Panas, S. 1998. A fuzzy vessel tracking algorithm for retinal images based on fuzzy clustering. IEEE Trans. Med. Imag., vol. 17, pp. 263-273.

[7] Hoover, A. D., Kouznetsova, V. and Goldbaum, M. 2000. Locating blood vessels in retinal images by piecewise threshold probing of a matched filter response. IEEE Trans. On Medical Imaging, vol. 19, no. 3, pp. 203-210.

[8] Gonzalez, R.C. Woods, R. E. 2008. Digital Image Processing. Pearson Education. Third Edition, pp. 627687.

[9] Joshi, S. and Karule, P. T. 2012. Retinal Blood Vessel Segmentation: International Journal of Engineering and Innovative Technology (IJEIT) Vol. 1, Issue 3.

[10] Salem, N. M. and Nandi, A. K. 2008. Unsupervised Segmentation of Retinal Blood Vessels using a Single
Parameter Vesselness Measure. Sixth Indian Conference on Computer Vision, Graphics \& Image Processing, IEEE Xplore, pp. 528-534.

[11] Staal, J. J., Abramoff, M. D., Niemeijer, M., Viergever, M. A. and Ginneken, B. V. 2004. Ridge-Based Vessel Segmentation in Colour Images of the Retina. IEEE Transactions on Medical Imaging, vol. 23, no. 4, pp. 501- 509 .

[12] Jiang, X. and Mojon, D. 2003. Adaptive Local Thresholding by Verification Based Multi-threshold Probing with Application to Vessel Detection in Retinal Images. IEEE Trans. Pattern Anal. Machine Intell., vol. 25, pp. 131-137.

[13] Zana, F. and Klein, J. C. 2001. Segmentation of Vessellike Patterns Using Mathematical Morphology and Curvature Evaluation. IEEE Trans. on Medical Imaging, vol. 10, no. 7, pp. 1010-1019.

[14] Samanta, S., Saha, S. K. and Chanda, B. 2011. A Simple and Fast Algorithm to Detect the Fovea Region in Fundus Retinal Image. Second International Conference on Emerging Applications of Information Technology, IEEE Xplore, pp. 206 - 209.

[15] Wang, Z., Bovik, A. C., Sheikh, H.R. and Simoncelli, E. P. 2004. Image quality assessment: from error visibility to structural similarity. IEEE Transactions on Image Processing, vol. 13 Issue 4, pp. 600-612 ( April 2004)

[16] DRIVE: Digital Retinal Images for Vessel Extraction http://www.isi.uu.nl/Research/Databases/DRIVE/ as on June 30 (2012).

[17] Abdou, I. E. and Pratt, W. K. 1979. Quantitative design and evaluation of enhancement/thresholding edge detectors. In Proceedings of the IEEE Conference. 67(5). 753-763.

[18] Kundu, A. and Chatterjee, R. K. 2012. Retinal vessel segmentation using Morphological Angular Scale-Space. 2012 Third International Conference on Emerging Applications of Information Technology 